\title{
Quantitative Analysis in Nuclear Medicine Imaging
}

\author{
H. Zaidi, ed.
}

New York, NY: Springer, 2006, 538 pages, $\$ 125$

Quantitative Analysis in Nuclear Medicine Imaging is an in-depth review of single-photon and PET nuclear medicine image reconstruction, correction, modeling, and analysis techniques, with a particular emphasis on quantitative imaging for both diagnostic and therapeutic (i.e., internal radionuclide dosimetric) purposes. The book is highly technical and uses numerous complex and multidimensional integral, differential, statistical, and trigonometric equations and formulas to describe a wide spectrum of imaging problems and analysis methods. Thus, in my opinion, this book is intended primarily for physicists, physicians, imaging scientists, and graduate students who wish to obtain a high level of knowledge of image processing and analysis methods applied to quantitative nuclear medicine imaging in diagnosis and therapy.

The editor, Dr. Zaidi, being either the first author or the coauthor of 13 of the 18 chapters, appears to have a wealth of knowledge and expertise in many aspects of nuclear medicine imaging. In addition, he has drawn on the expertise of 17 coauthors who are well-known authorities in their own right in their respective fields. The book is nicely illustrated, containing 110 figures sprinkled throughout the 17 main chapters, and each chapter contains plenty of good and relevant references for those who wish to further investigate a particular topic or application.

The book, in general, reads as a forward progression from the front end (image acquisition) to the back end (quantitative analysis methods on acquired or processed images) of the imaging chain and can be logically divided into 5 major sections: instrumentation, image reconstruction and corrections, image registration and segmentation, modeling in nuclear medicine imaging, and image quantification. An overview of the current state of the art of nuclear medicine imaging instrumentation, covering both human and animal scanning, and including PET/CT and SPECT/CT, is presented in the first 2 chapters. These are followed by 6 chapters extensively detailing analytic (e.g., filtered backprojection) and iterative tomographic reconstruction methods and methods of compensating for collimator-detector resolution, attenuation, scatter, and partial-volume effects. Chapters 9 and 10 describe strategies to handle registration of images from multiple modalities (e.g., deformations, similarity measures) and nuclear medicine image segmentation problems (e.g., clustering, neural networks, deformable models). The fourth section

COPYRIGHT (c) 2007 by the Society of Nuclear Medicine, Inc. DOI: 10.2967/jnumed.107.042598 presents the application of modeling to nuclear medicine imaging; Chapter 11 discusses the use of Monte Carlo methods to model the nuclear medicine imaging process and radiation transport calculations for internal radionuclide dosimetry, and Chapter 12 provides a detailed description of tracer kinetic (compartmental) modeling analysis of dynamic PET images. The last section contains 5 chapters that address quantification of activity in nuclear medicine images. One chapter is dedicated to quantification of planar images (e.g., conjugate-view whole-body), with an emphasis on estimating activity versus time for internal radionuclide dose calculation purposes. Quantitative analysis of functional brain images (e.g., brain mapping), myocardial perfusion SPECT (e.g., segment scoring, left ventricular ejection fraction), and nuclear oncologic images (e.g., glucose metabolism, standardized uptake value) is presented in 3 subsequent chapters, and the final chapter in the section addresses the topic of image-based internal dosimetry and treatment planning for radionuclide therapy. (Missing from the text are discussions of well-established, basic planar quantification methods such as multiple gated acquisition ejection fraction, dynamic renal glomerular filtration rate and effective renal plasma flow, lung ventilationperfusion analysis, and gastric emptying. However, I do not view this omission as a significant weakness, as descriptions of those methods can be found in just about any good textbook covering the basics of nuclear medicine, and those methods were not the focus of this book.)

I found Quantitative Analysis in Nuclear Medicine Imaging to be an excellent and up-to-date reference on advanced quantitative analysis methods applied to both single-photon and PET imaging. This book fills an information gap in this ever-evolving and advancing field of nuclear medicine and would serve as a useful adjunct to any basic physics text used in graduate-level nuclear medicine physics courses, which often cannot present all the topics included in this book with the same breadth and depth of coverage. The book could also be used as a stand-alone text for a course on advanced topics in nuclear medicine imaging. I recommend this book to all imaging scientists who are seriously interested or engaged in the application of quantitative processing and analysis techniques to nuclear medicine images.

William D. Erwin University of Texas M.D. Anderson Cancer Center Houston, Texas 